\title{
O lugar da pesquisa qualitativa na avaliação de políticas e programas sociais
}

\author{
Ana Maria Falsarella
}

Resumo: Este texto apresenta um estudo que explora e organiza ideias sobre tendências na avaliação de políticas e programas sociais. Parte dos três primeiros estágios da teoria da avaliação social (SHADISH, COOK; LEVINTON, 1995): $1^{\circ}$ Aplicação rigorosa de métodos científicos; $2^{\circ}$ Estudo dos fatores que influenciam a utilização da avaliação por decisores públicos; $3^{\circ}$ Síntese dos estágios anteriores e análise de seus pontos fortes e fracos. A seguir, apresenta a quarta geração ou estágio das teorias cuja base é o paradigma interpretativo (GUBA; LINCOLN, 1989). Destaca que a pesquisa qualitativa, no decorrer do tempo, ganha espaço no universo da pesquisa social ao reconhecer a influência das relações intersubjetivas e dos valores das pessoas envolvidas. Conclui sobre a complementaridade de métodos quantitativos e qualitativos.

Palavras-chave: Avaliação social. Políticas e programas sociais. Pesquisa qualitativa. Paradigma interpretativo.

The place of qualitative research in social policies and programs evaluation

Abstract: The text explores and organizes ideas on trends in the evaluation of social policies and programs. It starts with the presentation of the first three stages of the theory of social evaluation (SHADISH, COOK and LEVINTON, 1995): (1) Application of rigorous scientific methods, (2) Study of factors influencing the use of evaluation by decision makers; (3) Summary of previous stages and analysis of its strengths and weaknesses. In continuation, it presents the fourth generation of theories whose basis is the interpretive paradigm (GUBA and LINCOLN, 1989). It points out that qualitative research, over time, gains space in the universe of social research to recognize the influence of interpersonal relations and values of the people involved. It concludes discussing the complementarity of quantitative and qualitative methods.

Keywords: Social evaluation. Policies and social programs. Qualitative research. Interpretative paradigm. 


\section{Introdução}

A pesquisa qualitativa em suas origens mais remotas está associada ao romantismo e ao idealismo do final do século XIX, quando surge a reivindicação por uma metodologia mais autônoma e compreensiva para as "ciências do mundo da vida" (CHIZZOTTI, 2003, p. 224) a partir de estudos que, recorrendo a registros e documentações de época, descrevem as precárias e adversas condições de vida de trabalhadores rurais e urbanos franceses e ingleses no início da industrialização. Já no início do século XX, pesquisadores da área da antropologia colocavam em xeque o fato de os estudos nas ciências humanas se valerem do mesmo entendimento teórico das ciências exatas, ou seja, de que só no mundo objetivo seria possível recolher e verificar dados por meio da empiria. A pesquisa qualitativa passa então a se encaminhar na direção de obter um status de estudo tão rigoroso quanto o da pesquisa quantitativa.

É somente nas décadas de 1970 e 1980 que a consolidação de três fatores vai impulsionar o efetivo reconhecimento da pesquisa qualitativa nos meios acadêmicos. O primeiro é a gradativa onipresença de computadores possibilitando rapidez e maior racionalização na organização das informações. O segundo é o surgimento de inúmeras revistas científicas com enfoque mais qualitativo. $\mathrm{O}$ terceiro tem a ver com a força que ganham na sociedade questões de identidade (raça, classe social, gênero, minorias), que exigem pesquisa e respectiva escrita cada vez mais reflexiva.

Ao longo da década de 1990, na pesquisa qualitativa, a visão do pesquisador como observador passivo vai sendo gradativamente substituída por uma visão mais participativa e o método misto ou combinado de abordagem (quali-quantitativo) passa a ser utilizado por pesquisadores das ciências sociais, quebrando a ideia da incompatibilidade entre métodos quantitativos e métodos qualitativos.

Em síntese, a avaliação de programas surgiu, de forma sistemática, a partir da aplicação de métodos de pesquisa científica aos problemas sociais. Ao longo do tempo, alguns fatores contribuíram para aumentar a demanda de avaliação e modificar essa visão inicial. Dentre eles, a necessidade de estabelecer prioridades e de contemplar programas mais importantes. Para que determinados programas fossem mantidos, era preciso julgar sua eficácia e eficiência. Além do mais, a crescente participação da sociedade civil nas discussões sobre a destinação de recursos públicos aumentou a pressão por resultados e transparência (CALMON, 1999).

Chega-se, assim, à moderna teoria de avaliação de programas sociais, que articula teoria e questões essenciais ligadas aos próprios programas que estão 
sendo avaliados, considerando múltiplos olhares dos envolvidos (BAUER; NOVAES, 2012).

\section{Os três primeiros estágios de evolução da teoria da avaliação social}

Segundo Shadish, Cook e Levinton (1995), as teorias de avaliação de políticas e programas sociais evoluíram em três estágios ou gerações distintas. Segundo Lincoln (2005), a quarta geração é sucessora dos três modelos anteriores, mas considera premissas e operações distintas, principalmente a intensa participação dos envolvidos, tanto no programa avaliado quanto na organização da avaliação (FALSARELLA; LOMBARDI, 2012).

\section{Primeiro estágio: busca do rigor científico}

O primeiro estágio surge ao longo da década de 60 e apresenta duas características fundamentais: a preocupação com a resolução dos principais problemas sociais e a aplicação rigorosa de métodos científicos. Destacam-se, nessa fase, os trabalhos de Michael Scriven e Donald Campbell.

As contribuições de Scriven foram marcantes em vários aspectos. Um deles está relacionado ao campo normativo da avaliação de programas. Para ele, toda avaliação é claramente valorativa, já que, qualquer que seja o objetivo, o que se procura é analisar a qualidade de um programa. Ele também propunha que um programa poderia ser avaliado de forma objetiva, inclusive no que tange ao seu mérito, a partir de fatos empíricos. Portanto, "a avaliação deve gerar, como conclusão, exatamente o que os cientistas sociais, por longo tempo, julgaram ser ilegítimo: um julgamento de valor, mérito. Esse é o significado maior do ponto de vista científico e filosófico da avaliação." (SCRIVEN apud SHADISH; COOK; LEVINTON, 1995, p. 75).

Scriven criou, ainda, uma tipologia para a teoria de avaliação e introduziu alguns conceitos, noções e termos fundamentais, tais como:

1. Formative evaluation, ou avaliação formativa, realizada com o intuito de gerar feedback para melhoria de um programa;

2. Summative evaluation, ou avaliação somativa, que visa exclusivamente julgar o mérito do programa;

3. Goal-free evaluation, ou avaliação sem consideração dos objetivos, na qual os objetivos preestabelecidos do programa não são considerados como parâmetros de avaliação; 
4. Cost-free evaluation, ou avaliação sem custos, que considera que a avaliação de um programa deve ocorrer apenas quando os benefícios por ela gerados excederem os custos da sua realização;

5. Metaevaluation, ou meta-avaliação, que implica na consideração de avaliações ou análises de avaliações realizadas anteriormente.

Os estudos de Campbell e Stanley (apud SHADISH; COOK; LEVINTON, 1995) por sua vez, influenciaram de forma marcante o campo da avaliação no que tange à utilização de métodos e técnicas quantitativas de pesquisa científica em estudos de caráter social. Para ele, as mudanças sociais deveriam estar fundamentadas na experimentação e a sociedade poderia ser convertida em uma sociedade de experimentos (experimenting society). Consequentemente, a efetividade dos programas seria passível de estudo por meio de experimentos naturais, mediante a utilização de técnicas de pesquisa experimentais ou quase-experimentais ${ }^{1}$.

Uma característica fundamental desse primeiro estágio de evolução da teoria da avaliação social é a preocupação com a validade dos instrumentos de pesquisa e com a evitação de vieses ou ambiguidades na interpretação dos experimentos. Acreditava-se que a avaliação, se adequadamente conduzida, constituiria importante instrumento de feedback, e que os seus resultados seriam automaticamente utilizados pelos formuladores de políticas, decisores e administradores.

\section{Segundo estágio: ênfase na utilização e no pragmatismo}

O segundo estágio inicia-se na década de 1970 e seu aspecto mais relevante é a preocupação com as questões do pragmatismo e do incremento do uso da avaliação nas tomadas de decisão sobre a concepção, alteração ou continuidade de programas sociais. Destacam-se, nessa segunda fase, os trabalhos de Carol Weiss, Joseph Wholey e Robert Stake.

Os estudos desses autores surgem da percepção de que, na prática, as informações geradas pelas avaliações nem sempre eram utilizadas adequadamente pelos formuladores de políticas e não conduziam necessariamente ao aperfeiçoamento dos programas. Em alguns casos, por exemplo, avaliações

1 A pesquisa experimental é um método de investigação que busca estabelecer relações de causa-efeito entre as variáveis investigadas, de modo que uma variável independente é manipulada para julgar seu efeito sobre uma variável dependente. Na pesquisa quase-experimental, o controle das variáveis é mais difícil. O desafio do experimentador é delinear um ambiente o mais próximo possível do mundo real, enquanto procura controlar, da melhor forma, algumas variáveis que podem interferir na validade da investigação. 
com padrões científicos deficientes eram consideradas aceitáveis pelos que as utilizavam. Em outros, a preocupação excessiva com o rigor metodológico e científico dos trabalhos de avaliação demandava tamanha duração que impossibilitava que as informações estivessem disponíveis a tempo de subsidiar tomadas de decisões.

Assim, o foco dos trabalhos do segundo estágio converge para a questão do aumento da utilização das informações e recomendações provenientes dos estudos de avaliação na melhoria dos resultados dos programas. Isso porque, em primeiro lugar, considerava-se que a utilização direta ou instrumental da avaliação não ocorreria de forma espontânea. Então, o avaliador deveria selecionar as necessidades dos diversos interessados (stakeholders) na avaliação e seus resultados e reputá-las como variáveis importantes. Em segundo lugar, preconizava-se que as informações decorrentes da avaliação também poderiam ser utilizadas conceitualmente (enlightment), contribuindo para o entendimento de questões e problemas relativos ao programa e gerando novas ideias e perspectivas.

Foi no segundo estágio que surgiu o debate sobre a utilização de técnicas quantitativas versus técnicas qualitativas na avaliação de programas. Tal debate decorria do pluralismo de métodos, conceitos e tipos de avaliação, bem como da ausência de consenso entre os teóricos sobre as melhores práticas a serem utilizadas neste universo de diversidade metodológica. Alguns teóricos qualitativos, como Egon Guba e Yvonna Lincoln (que são destacados mais adiante como representantes da quarta geração das teorias de avaliação social), propõem que o estudo de caso é um método tão bom quanto outros métodos de inferência causal e, em muitos casos, é preferível porque permite responder a uma gama mais ampla de questões. Passa-se a entender que os experimentos são ferramentas muito limitadas na pesquisa social, a qual demanda maior amplitude de métodos. Assim, no segundo estágio, as discussões giram em torno da questão: Quais métodos produzem respostas de mais alta qualidade para determinados tipos de questões?

\section{Terceiro estágio: integração de técnicas de acordo com o contexto}

O terceiro estágio consiste basicamente na síntese dos trabalhos dos dois estágios anteriores. Os teóricos mais importantes desse estágio são Lee J. Cronbach e Peter Rossi. Estes dois autores desenvolveram teorias que integram e legitimam as técnicas, os métodos e as preocupações das teorias do primeiro e segundo estágios, mas de forma condicional. Para os autores, a escolha 
de uma determinada prática de avaliação depende, dentre outras coisas, das circunstâncias, do contexto e do propósito da avaliação.

Há consenso, no terceiro estágio, de que os programas são afetados politicamente, caracterizam-se por diversidade epistemológica e metodológica, e possuem prioridades múltiplas, o que impossibilita a existência de uma prática universal e ideal de avaliação de programas sociais. Cronbach, apoiando-se em Carol Weiss, James March e Charles Lindblom, associa políticas e programas aos contextos sociais em que são desenvolvidos, sendo a avaliação considerada parte intrínseca do estudo das políticas, não algo à parte. Assim, nenhum programa merece ser avaliado a não ser que se procure averiguar como os grupos interessados o aprovaram, influenciaram sua concepção, participaram da sua implementação, o monitoraram e ajudaram a modificá-lo. Como afirmam Shadish, Cook e Levinton (1995), "todas as teorias de avaliação requerem fundamentação nas realidades política e administrativa, que criam, mantêm e restringem cada programa" (p. 351).

A principal contribuição de Rossi foi sistematizar os trabalhos realizados nas décadas de 1960, 1970 e 1980. Como parte desse esforço, ele propõe três conceitos fundamentais:

1. Comprehensive evaluation, ou avaliação abrangente, que surgiu da constatação de que, ao longo dessas décadas, foram adotadas práticas de avaliação centradas ora na formulação, ora na implementação, ora na análise dos resultados finais dos programas. Tal diversidade gerou, além da especialização, práticas de avaliação distintas e muitas vezes incompatíveis. Rossi reafirmou a importância dessa diversidade e especialização, mas propôs que fossem integradas. Uma avaliação deve, sempre que possível, envolver atividades relacionadas com todas as fases de um programa, quais sejam: a sua concepção (design); implementação (implementation); e a verificação (assessment) da sua efetividade e eficiência (program utility).

2. Tailored evaluation, ou avaliação sob medida, surgida da consideração de que nem sempre a realização da avaliação abrangente é factível face às restrições de tempo e recursos. Rossi propõe, então, o conceito de avaliação sob medida, na qual as práticas de avaliação variam em função do estágio de desenvolvimento do programa: cada avaliação deve ser adequada (tailored) ao programa a que se destina. As tarefas dos avaliadores dependem do estágio em que se encontra o programa e das necessidades e interesses dos stakeholders envolvidos na avaliação 
(formuladores de políticas, administradores e grupos financiadores). Além disso, as avaliações variam dependendo de o programa ser novo, já estabelecido ou estabelecido necessitando de ajustes.

As avaliações de programas novos geralmente são realizadas para determinar o seu impacto e a sua eficiência e requerem que o staff (quadro dos dirigentes ou grupo/equipe de assessoria de uma organização) e os financiadores repensem alguns ou todos os aspectos do programa (objetivos, características da população-alvo e medidas de resultados). As avaliações de programas já estabelecidos na maior parte das vezes se destinam a verificar até que ponto os objetivos do programa estão claros e são relevantes para os interessados, a averiguar se o programa está sendo executado conforme o programado e se tem alcançado o alvo apropriado. As avaliações direcionadas ao ajuste ou adequação dos programas já estabelecidos buscam redefinir a população alvo, modificar as formas de intervenção ou o próprio programa para aumentar a sua efetividade e eficiência.

1. Theory-driven evaluation, ou avaliação direcionada pela teoria, que surge de outra constatação feita por Rossi, a de que inúmeras práticas de avaliação são feitas de maneira ateórica, ou seja, ignorando as teorias subjacentes à formulação do programa avaliado. Isso parece ser paradoxal, visto que os programas governamentais são elaborados a partir de referenciais teóricos específicos que guiam e orientam não apenas o diagnóstico dos problemas a serem resolvidos, mas também as soluções mais adequadas. Nesse sentido, Rossi propõe que o avaliador leve em consideração as bases teóricas que foram utilizadas na concepção e implementação do programa, examinando a sua adequação e os seus limites, e tornando, assim, a prática da avaliação integrada aos debates teóricos e conceituais correntes.

Em síntese, tanto Rossi quanto Cronbach discutem as potencialidades e as fragilidades dos diferentes métodos e técnicas de avaliação propostos por diferentes teóricos e especificam, de maneira contingencial, sob quais circunstâncias é interessante ou não sua utilização. Eles não desconsideram a contribuição e o valor das soluções apresentadas por esses teóricos para os problemas enfrentados pelos avaliadores, mas propõem o uso de múltiplos métodos sempre que possível, bem como a combinação de técnicas quantitativas e qualitativas. 


\section{A quarta geração das teorias de avaliação}

No texto Fourth-Generation Evaluation, Yvonna Lincoln (2005) explora o que ela própria e Egon Guba (GUBA; LINCOLN, 1989) chamam de quarta geração das teorias de avaliação de programas e políticas sociais, que parte de pressupostos diferentes das três vertentes anteriores e conduz a distintos modos de operação na situação a ser avaliada. A premissa inicial é que os diversos agentes envolvidos na avaliação reagem não somente à realidade física ou tangível, mas também às suas próprias construções sócio-psicológicas sobre determinada situação. Apoia-se, assim, em um paradigma interpretativo. $\mathrm{Na}$ literatura sobre o tema, este paradigma aparece com diferentes termos, mas que guardam o mesmo sentido: perspectiva relativista da realidade, estudo naturalístico, metodologia interativa, pesquisa qualitativa, metodologia pluralista-construtivista ${ }^{2}$. Os teóricos deste estágio defendem que o objetivo da avaliação de programas e políticas sociais é desenvolver e aprofundar o conhecimento de uma dada situação em um dado contexto.

Para os propositores da quarta geração, um programa social só tem sentido quando desenvolvido e analisado em estreita relação com o contexto e com possíveis mudanças sociais que pode provocar. Além disso, como a atribuição de valores é inerente ao ser humano, é fundamental que os valores dos envolvidos sejam considerados. Por outro lado, entende-se que o conhecimento coletivamente construído na ação grupal leva à mudança social.

Técnicas e métodos da quarta geração aparecem relacionados ao conceito de círculo hermenêutico-dialético (CHD) ${ }^{3}$ proposto principalmente por Guba e Lincoln (1989), metodologia de pesquisa apresentada por estes autores como a mais capaz de dar conta de uma interpretação aproximada da realidade, pois coloca a fala dos atores em seu contexto de modo a entendê-la no seu interior

2 Neste tipo de pesquisa, os dados coletados são classificados em padrões, que se tornam a base primária para a organização e o relato dos resultados, sendo que as formas de observação podem variar muito de uma configuração para outra. Uma forma bastante utilizada é a observação participante, considerada uma estratégia de aprendizagem reflexiva [e não apenas um método de coleta de informações] em que os pesquisadores geralmente tornam-se membros de um grupo e adotam papéis conforme tal configuração com o objetivo de adquirir maior compreensão do que configura a cultura do grupo, suas práticas cotidianas, motivações e emoções. Argumenta-se que a capacidade dos pesquisadores para compreender as experiências da cultura pode ser inibida se observar sem participar.

3 A metodologia da chamada quarta geração ou metodologia pluralista-construtivista (GUBA; LINCOLN, 1989) apresenta a técnica do círculo hermenêutico-dialético [CHD] como um procedimento dinâmico, com constante interação entre as pessoas por intermédio do permanente vai e vem no processo de realização das entrevistas, discussões e observações, supondo constantes diálogos, críticas, análises, construções e reconstruções coletivas, o que ajuda no aprofundamento das reflexões coletivas sobre a percepção da realidade e permite chegar o mais próximo possível do consenso sobre ela. A utilização do CHD merece uma constante atenção tanto no processo de coleta de dados, como na fase de interpretação das informações; além disso, é importante o emprego da pré-análise das conclusões pelos entrevistados ou grupos, procedimento que minimiza a subjetividade do pesquisador e facilita a elaboração da síntese final. 
e no campo da especificidade histórica e totalizante em que é gerada. Fala-se em interpretação aproximada da realidade porque, mesmo de uma perspectiva sistêmica, não é possível chegar a uma interpretação total da realidade, em função do limite de nossa capacidade de objetivação.

Podemos destacar como ideias centrais das teorias de quarta geração, em primeiro lugar a oposição à investigação positivista que pressupõe uma relação direta de causa e efeito entre os fenômenos. Em segundo, a incidência do foco sobre o significado humano da vida social e sua clarificação e exposição por parte do investigador. Em terceiro, a visão de que o mundo real vivido é uma construção dos atores sociais que, em cada momento e espaço, constroem o significado social dos acontecimentos e fenômenos do presente e reinterpretam o passado. Por fim, a relevância dada à compreensão, à explicação e à interpretação como atividades humanas por excelência que permitem às pessoas conhecer-se a si próprias e às outras.

São conceitos básicos com os quais trabalham as teorias de quarta geração:

a) Metodologia interativa: métodos e técnicas de pesquisa são selecionados e utilizados segundo a realidade empírica em estudo (realidade, contexto) e de forma combinada com os participantes (atores sociais, stakeholders);

b) Realidade e contexto implicam em movimento, com fatos e fenômenos conectados e em mutação; a interpretação da dinâmica do processo em que se desenvolvem os fatos e fenômenos em estudo é feita por meio de estudos hermenêutico-dialéticos;

c) Um estudo hermenêutico-dialético consiste na interpretação da dinâmica da realidade dentro de uma visão sistêmica, buscando-se a compreensão da rede de interações, a qual vai se aprofundando durante a observação do fenômeno em estudo; vai-se percebendo o dinamismo dessas interações e relações na medida em que se percebe que o fenômeno que constitui o objeto de estudo é dinâmico, dialético, instável, em constante mudança.

d) Uma visão sistêmica implica compreender o processo de conhecimento como dinâmico e interligado, isto é, as partes só podem ser compreendidas dentro do todo. Busca-se, concomitantemente, entender, interpretar e analisar fatos e fenômenos à luz de teorias pertinentes.

Além desses conceitos básicos, podem ser destacadas as seguintes características nas teorias de quarta geração: 
- Definição expandida de stakeholder, que envolve não só formuladores e gerenciadores do programa, mas também os destinatários e outros membros da comunidade que têm algum interesse nos resultados da avaliação;

- Relação interativa, interdependente e intersubjetiva entre o avaliador e os stakeholders, o que leva a uma posição comum, mais rica e diversificada, contrapondo-se aos antigos modelos em que os stakeholders eram vistos apenas como fontes de dados;

- Participação intensa dos stakeholders na determinação do percurso que a avaliação vai trilhar e na seleção e identificação das ações que serão utilizadas;

- Entendimento de que a coleta e análise de dados sobre as construções sociais têm a mesma relevância que a coleta e análise das informações mensuráveis (números, escores);

- Utilização de repertório metodológico expandido, adicionando uma série de métodos qualitativos ao arsenal estatístico existente.

As teorias da quarta geração não têm subjacente uma mas diversas epistemologias, influenciadas pela diversidade de perspectivas, interpretações e percepções dos agentes/indivíduos envolvidos sobre uma mesma situação. Em contraposição à lógica positivista, partem de axiomas filosóficos da fenomenologia e da teoria crítica. Da ótica fenomenológica (estudo da natureza da realidade), a realidade não é física e tangível, mas uma singular construção mental e social de indivíduos e grupos que emana do senso do fazer, ou seja, dos sentidos imputados pelos seres humanos às atividades cognitivas. Sendo assim, não há caminho único; pelo contrário, diferentes construções se ajustam holisticamente, reforçando-se e apoiando-se mutuamente. Da perspectiva da teoria crítica, as diversas realidades e situações são passíveis de serem tratadas historicamente, podendo ser alteradas ou redirecionadas para promover maiores níveis de participação da sociedade civil, de justiça social e democracia.

Quanto ao papel do investigador, entende-se que é impossível estabelecer uma separação nítida entre ele e o objeto de estudo, pois toda a investigação apresenta marcas de quem a realiza. Portanto, é impossível ao observador posicionar-se apenas externamente à realidade do contexto. Em vista disso, a intersubjetividade, resultante da interação e da colaboração entre o investigador e os stakeholders passa a ser reconhecida como fator interveniente na 
pesquisa. O investigador vai construindo, por meio de análises sucessivas, uma teoria que explique o fenômeno que está estudando na medida em que desenvolve seu estudo, começando por um conjunto empírico de dados. Uma vez que o investigador tem uma relação muito próxima com os participantes, deve-se dar muita atenção a questões de ordem ética, que dizem respeito à responsabilidade na relação estabelecida com os sujeitos do estudo. Assim, alguns princípios éticos devem ser seguidos pelo pesquisador:

a) Garantir o consentimento informado dos participantes, resultante de uma informação clara por parte do investigador quanto aos objetivos e aos procedimentos que pensa utilizar, ou seja, as regras do jogo devem ser claras e negociadas; dados que não sejam do conhecimento dos participantes e não tenham seu consentimento prévio não devem ser coletados;

b) Pensar nas possíveis implicações e situações embaraçosas, decorrentes da publicação do estudo, a que estão sujeitos os participantes; os limites de acesso aos dados devem ser discutidos e negociados [com especial cuidado com questões de foro íntimo e de privacidade dos participantes], podendo o investigador recorrer ao anonimato e a pseudônimos;

c) Evitar juízos sobre o objeto de estudo com base nos próprios valores, sendo que o risco de que isso aconteça é grande porque todo pesquisador tem suas próprias concepções; embora ao investigador não seja possível assumir uma postura avaliativa exterior totalmente isenta, ele deve procurar interpretar e compreender os significados das informações para os participantes.

Vale ressaltar que uma pesquisa qualitativa não tem nenhum tipo de esquema fechado, tudo dependendo do fenômeno estudado em contexto. Ela envolve uma multiplicidade de métodos de investigação, empregados em diversas disciplinas acadêmicas, principalmente nas ciências sociais, sendo que o estudo de caso é considerado o método típico do paradigma interpretativo, bastante utilizado quando se pretende compreender e analisar determinados problemas ou situações particulares em maior profundidade. Basicamente, o estudo de caso consiste na investigação de uma dada realidade no seu contexto real destacando o que nela há de essencial, único e característico e na descrição analítica intensiva e globalizante da situação ou fenômeno observado. A coleta de dados pode incluir entrevistas, discussões em grupo e observações [estruturadas, semiestruturadas ou livres]. Os registros podem ser feitos por meio 
de anotações sobre fenômenos e reflexões feitas em campo [diários reflexivos, diários de campo, diários de bordo], textos variados, imagens, gravações, filmagens e leitura e análise de documentos e outros materiais. Mas nunca será possível conhecer tudo sobre um caso. Cabe ao investigador decidir até onde deve ir, qual o nível de profundidade do conhecimento a que pretende chegar, de forma a ser-lhe possível atingir os objetivos a que se propõe.

\section{Concluindo}

No decorrer do texto, buscamos expor como a pesquisa qualitativa gradativamente ganhou espaço no universo da pesquisa social. Pesquisadores qualitativos visam a uma compreensão aprofundada do comportamento humano e das razões que governam tal comportamento, o que não é possível com a utilização meramente quantitativa de dados.

Os defensores da pesquisa qualitativa apontam inúmeras vantagens em sua utilização. A mais relevante é que, por seu intermédio, pode-se investigar o porquê e o como das situações e não apenas o que, onde e quando os fenômenos acontecem. Deste modo justificam que investigar universos menores e mais focados é tão ou mais importante do que fazê-lo com grandes amostras. Assim, mais recentemente, a perspectiva convencional, que entende a abordagem qualitativa produz informações apenas sobre casos particulares estudados e pode, no limite, ser usada como apoio empírico para hipóteses de investigação, vem sendo substituída por uma visão mais ampla. Entende-se que as conclusões de um estudo qualitativo, mesmo não sendo replicáveis a outras situações, podem ser consideradas indicadores de tendências, sendo possível, inclusive, estabelecer algumas generalizações, respeitados os limites convencionados.

De qualquer modo, a atual tendência na avaliação de políticas e programas sociais é a realização de estudos de caráter quali-quantitativo (misto ou combinado), entendendo-se que abordagens qualitativas e quantitativas não são incompatíveis, mas sim complementares. Dados quantitativos, sistematizados, são iluminados por componentes qualitativos, assegurando uma melhor compreensão dos fenômenos.

\section{Referências}

BAUER, A.; NOVAES, G. Curso: Avaliação de políticas e programas educacionais. São Paulo: Fundação Carlos Chagas (FCC), fev./ jul. 2012. 
CALMON, K. A avaliação de programas e a dinâmica de aprendizagem organizacional. Planejamento e Políticas Públicas, Brasília, n. 19, 1999.

CHIZZOTTI, A. A pesquisa qualitativa em ciências humanas e sociais: evolução e desafios. Revista Portuguesa de Educação. Braga-PT, v. 16, n. 2, p. 221-236, 2003.

FALSARELLA, A. M.; LOMBARDI, M. R. A abordagem subjacente ao texto Fourth-generation evaluation em relação às cinco dimensões propostas por Shadish, Cook e Levinton para descrever teorias de avaliação. Trabalho final. (Curso Avaliação de políticas e programas educacionais). São Paulo: FCC, jul. 2012.

GUBA, E.; LINCOLN, Y. Fourth generation evaluation. Califórnia-USA: Sage, 1989.

LINCOLN, Y. Fourth-Generation Evaluation. In: MATHISON, S. (Ed.). Encyclopedia of evaluation. California-USA: Sage, 2005. p. 161-164.

SHADISH, W.; COOK, T.; LEVINTON, L. Foundations of program evaluation: theories of practice. California-USA: Sage, 1995.

Ana Maria Falsarella - Centro Universitário de Araraquara São Paulo | SP | Brasil. Contato: anafalsarella@gmail.com

Artigo recebido em 20 de julho de 2013 e aprovado em 27 de novembro de 2013. 
